\title{
EFECTO DE LA MADURACIÓN in vitro DE OOCITOS BOVINOS CON SUERO FETAL BOVINO SOBRE SU ACTIVIDAD MITOCONDRIAL POST-DESVITRIFICACIÓN
}

\author{
THE EFFECT OF in Vitro MATURATI ON OF BOVINE OOCITES \\ WITH FETAL BOVI NE SERUM ON THEI R MITOCHONDRI AL \\ ACTI VITY FOLLOWI NG DEVITRI FICATION
}

\author{
Giovanni Restrepo B ${ }^{1 *}$, M.Sc, Neil Vásquez², M.Sc. \\ ${ }^{1}$ Politecnico Colombiano Jaime Isaza Cadavid, Facultad de Ciencias Agrarias, Carrera 48 \\ $N^{\circ}$ 7-151, Medellín, Colombia. ${ }^{2}$ Universidad Nacional de Colombia, Departamento de \\ Biociencias, Calle 59A No 63-20 Medellín, Colombia. *Correspondencia: \\ grestrepo@elpoli.edu.co
}

Recibido: Julio 10 de 2008; Aceptado: Diciembre 12 de 2008

\section{RESUMEN}

Objetivo. Evaluar el efecto de la maduración in vitro (MIV) con suero fetal bovino (SFB) sobre la actividad mitocondrial (potencial de membrana) de oocitos bovinos desvitrificados. Materiales y métodos. Se recolectaron oocitos bovinos a partir de ovarios post-mortem, para su posterior MIV en medio Sintetic Oviductal Fluid Amino Acids (SOFaa) en tratamientos con y sin SFB. Posteriormente, fueron destinados para su criopreservación mediante vitrificación en Open Pulled Straw (OPS). Los oocitos fueron desvitrificados y evaluados para su potencial de membrana mitocondrial mediante el uso del colorante fluorescente JC- 1 . Los datos fueron analizados mediante la prueba t de Student y un análisis de varianza. Resultados. Los resultados evidenciaron una diferencia estadística $(p<0.05)$ entre los tratamientos de MIV para los niveles cuantificados de potencial de membrana mitocondrial de los oocitos desvitrificados, con promedios de $39.8 \pm 2.38 \%$ y $50.3 \pm 2.59 \%$ para MIV con y sin suero respectivamente. No se encontró un efecto del suero en la MIV, sobre la distribución de mitocondrias de alto potencial de membrana, sin embargo, se observó diferencia estadística $(p<0.05)$ para este parámetro entre los oocitos frescos y desvitrificados. Conclusiones. La presencia de SFB en la MIV de oocitos bovinos, ejerció un efecto deletéreo sobre el potencial de membrana mitocondrial posterior a la criopreservación, y esta última modifica las proporciones de los patrones de distribución de mitocondrias de alto potencial de membrana de los oocitos bovinos madurados in vitro.

Palabras clave: Oocitos, bovinos, maduración, criopreservación, vitrificación, membrana, mitocondria. 


\section{ABSTRACT}

Objective. To evaluate the effect of in vitro maturation (IVM) with fetal calf serum (FCS) on the mitochondrial activity (membrane potential) of devitrified bovine oocytes. Materials and methods. Bovine oocytes were collected from post-mortem ovaries and matured in vitro in SOFaa medium with and without FCS. The oocytes were cryopreserved by OPS vitrification. Devitrified oocytes were evaluated for their mitochondrial membrane potential by J C- 1 dye staining. Statistical evaluations were done using Student's t-test and analysis of variance. Results. There was a statistically significant difference $(p<0.05)$ between IVM treatments for the mitochondrial membrane potential levels of devitrified oocytes, with averages of $39.8 \pm 2.38 \%$ and $50.3 \pm 2.59 \%$ for MIV with and without serum, respectively. There was no IVM with serum effect over the distribution of high mitochondrial membrane potential; nevertheless there was a statistically significant difference $(p<0.05)$ in this parameter between fresh and devitrified oocytes. Conclusions. FCS in the IVM has a deleterious effect on mitochondrial membrane potential after cryopreservation and cryopreservation alters the proportions of distribution patterns of high activity mitochondria in in vitro matured bovine oocytes.

Key words: Oocytes, bovine, maturation, criopreservation, vitrification, membrane, mitochondrial.

\section{NTRODUCCIÓN}

La criopreservación de oocitos bovinos es valiosa al favorecer la disponibilidad de material genético para los procedimientos de producción de embriones, ingeniería genética y preservación de germoplasma (1), sin embargo, el éxito de esta tecnología ha sido deficiente (2), debido a la alta sensibilidad y vulnerabilidad de los oocitos cuando son expuestos a la hipotermia $(3,4)$, lo que se refleja en las bajas tasas de sobrevivencia, fertilización y desarrollo a partir de oocitos criopreservados (5).

Numerosos reportes han sugerido que la criopreservación altera la membrana plasmática (6), los microtúbulos y la organización del citoesqueleto (7), las mitocondrias (8), los cromosomas (9) y la zona pelúcida, a través de daños provocados por la formación de cristales de hielo, choques osmóticos, el efecto tóxico de los crioprotectores, la concentración intracelular de electrolitos y el enfriamiento (10). Un importante parámetro ligado a la reducción de la criotolerancia de oocitos y embriones es su alto nivel de contenido lipídico $(11,12)$, del cual se ha señalado como responsable a la adición de suero en los medios utilizados para la producción in vitro de embriones, a causa de una excesiva incorporación citoplasmática de lípidos $(1,13)$ que puede inducir la formación de cristales de hielo $(14,15)$, y además posiblemente conduce a un trastorno en el metabolismo de su población de mitocondrias $(16,17)$.

Algunas observaciones indican que las mitocondrias son funcionalmente trascendentes para los oocitos y los embriones tempranos $(18,19)$, ya que se sugiere una relación funcional entre la fosforilación oxidativa, la producción de ATP y el desarrollo embrionario $(20,21)$, y se ha propuesto que la pérdida de actividad mitocondrial (potencial de membrana) en oocitos puede contribuir al bajo desarrollo embrionario durante la producción in vitro (22-24), así como a las bajas tasas de preñez post-transferencia (25). Además, estudios recientes fijan su atención en la forma como la criopreservación afecta la mitocondria, su actividad, la generación de ATP derivada de su función metabólica, y el desarrollo embrionario in vitro $(11,17,26)$. Han sido descritas redistribuciones mitocondriales en oocitos y embriones en los estadios tempranos de desarrollo preimplantacional de varias especies $(18,23$, 
27). En bovinos la principal redistribución de las mitocondrias ocurre durante la maduración del oocito, y parece estar correlacionada con el grado de desarrollo de competencia adquirido por el oocito $(18,28)$, pues como la mitocondria sintetiza ATP y sirve como una fuente interna de $\mathrm{Ca}_{2}{ }^{+}$, es posible que éstas se redistribuyan y se agreguen dentro del oocito para concentrar el ATP (29).

Teniendo como base un estudio preliminar en el cual se determinó la existencia de un efecto deletéreo de la suplementación de la maduración in vitro (MIV) con suero fetal bovino, sobre la actividad mitocondrial y el desarrollo embrionario in vitro de oocitos bovinos (30). Como método para la criopreservación de oocitos, se empleó la técnica de vitrificación en open pulled straw (OPS), la cual permitió disminuir los daños por enfriamiento y los efectos osmóticos y tóxicos, se aumentó la velocidad de los cambios de temperatura (hasta $20^{\circ} \mathrm{C} / \mathrm{min}$ ) y permitió la disminución de las concentraciones de crioprotectores (30).

El objetivo de este trabajo fue evaluar el efecto de la maduración in vitro (MIV) en presencia de suero fetal bovino, sobre la actividad mitocondrial de oocitos bovinos criopreservados.

\section{MATERI ALES Y MÉTODOS}

\section{Recolección de ovarios y complejos cúmulus-oocito. Los oocitos bovinos} fueron recuperados desde ovarios de hembras provenientes de la planta de faenado local, transportados en solución salina ( $\mathrm{NaCl} 0,9 \%)$ a $38^{\circ} \mathrm{C}$, y procesados en el laboratorio dentro de las tres horas posteriores a su recolección. Los folículos entre 2 y $6 \mathrm{~mm}$ fueron aspirados usando una jeringa de $5 \mathrm{ml}$ con una aguja calibre 18. Los complejos cúmulus-oocito (CCOs) y el fluido folicular recuperados fueron puestos en tubos cónicos plásticos de 50 $\mathrm{ml}$, dejándolos sedimentar durante 15 minutos a $37^{\circ} \mathrm{C}$ para realizar la recuperación posterior de la fracción precipitada, desde la cual se seleccionaron solamente los complejos con características de ooplasma homogéneo, mínimo tres capas compactas de células de la granulosa rodeando completamente al oocito, y morfología intacta. Los CCOs seleccionados fueron lavados en medio Hepes-TL suplementado con $0.2 \mathrm{mM}$ de piruvato de sodio (Sigma P5280) y $3 \mathrm{mg} / \mathrm{ml}$ de albúmina sérica bovina fracción V (Sigma A9647).

Maduración in vitro de oocitos. Se establecieron dos tratamientos de maduración in vitro de oocitos. Para el primer tratamiento, denominado de maduración sin suero, los CCOs fueron incubados en grupos de 10 por gota de medio de maduración, donde cada gota de $50 \mu \mathrm{l}$ consistió en medio de fluido oviductal sintético (SOF), suplementado con $1 X$ de medio esencial mínimo con aminoácidos no esenciales (MEMneaa, MEM Eagle Sigma M0268), 1X de medio esencial mínimo con aminoácidos esenciales (MEMeaa, BME Sigma B6766), $0.33 \mathrm{mM}$ de piruvato de sodio, $1.5 \mathrm{mM}$ de $D$ (+)-glucosa (Sigma G7021), $8 \mathrm{mg} / \mathrm{ml}$ de albúmina sérica bovina libre de ácidos grasos (BSA FAF, Sigma A6003), LH ( $10 \mu \mathrm{g} / \mathrm{ml}), \mathrm{FSH}$ $(10 \mu \mathrm{g} / \mathrm{ml})$, y $10 \mu \mathrm{l} / \mathrm{ml}$ de solución antibiótica (100X ICN 1674046, penicilina $10.000 \mathrm{UI} / \mathrm{ml}$; estreptomicina $10 \mathrm{mg} / \mathrm{ml}$; anfotericina $B$ $25 \mathrm{mg} / \mathrm{ml}$ ). Las gotas fueron cubiertas con aceite mineral (Sigma M8410) y preincubadas bajo condiciones de maduración por un mínimo de $3 \mathrm{~h}\left(38.5 \circ \mathrm{C}, 5 \% \mathrm{CO}_{2}\right.$, con $100 \%$ de humedad), luego los CCOs fueron transferidos a las gotas, para ser incubados por $24 \mathrm{~h}$ (38.5으, 5\% $\mathrm{CO}_{2}$ con $100 \%$ de humedad). Para el segundo tratamiento, denominado de maduración con suero, los CCOs fueron incubados igualmente en grupos de 10 por gota de medio, donde cada gota de $50 \mu \mathrm{l}$ de medio consistió en SOF suplementado de igual manera que el medio sin suero, más $10 \%$ de suero fetal bovino (SFB, Gibco BRL). Las gotas fueron cultivadas mediante el mismo procedimiento del tratamiento sin suero.

Criopreservación de oocitos. Para la fabricación de pajillas estiradas para la técnica de vitrificación en OPS, pajillas plásticas para $0.25 \mathrm{ml}$ de volumen fueron reblandecidas al apoyar su tercio central por 3 segundos sobre la esquina de una plancha térmica a más de $100^{\circ} \mathrm{C}$. Ambos extremos de la pajilla fueron estirados al ser extendidos 
en un plano horizontal hasta lograr en la porción central la reducción del diámetro interno y externo aproximadamente a la mitad de la medida original, con la consecuente disminución del grosor de la pared. Luego las pajillas se cortaron en la sección más delgada con un bisturí, para posteriormente ser esterilizadas utilizando luz ultravioleta y almacenadas hasta su uso.

Veinticuatro horas después de comenzar la maduración in vitro, los oocitos madurados con y sin suero fueron parcialmente desnudados por pipeteo y exposición a $0.1 \%$ de hialuronidasa hasta que sólo 2 a 4 capas de células del cúmulus permanecieron en su superficie. Los oocitos fueron transaferidos a un medio de equilibrio (EQ) compuesto por TCM-199 Hepes (Sigma M7528) con 20\% de SFB, durante 5 minutos a una temperatura de $39^{\circ} \mathrm{C}$. Luego fueron expuestos a $1 \mathrm{ml}$ de una primera solución de vitrificación (V1) compuesta por TCM- 199 Hepes con $10 \%$ de etilenglicol (EG, Sigma E9129), 10\% de dimetilsulfoxido (DMSO, Sigma D2650) y 20\% de SFB, durante 30 segundos a $39 \circ \mathrm{O}$. Los oocitos fueron luego transferidos a gotas de $20 \mu \mathrm{l}$ de una segunda solución de vitrificación (V2) compuesta por TCM-199 Hepes con $20 \%$ de EG, $20 \%$ de DMSO, $20 \%$ de SFB, y $0.5 \mathrm{M}$ de sacarosa, durante 30 segundos a $39^{\circ} \mathrm{C}$. Grupos de entre 3 a 5 oocitos fueron tomados con una pipeta automática en un volumen de $2 \mu \mathrm{l}$ de $\mathrm{V} 2$, para depositarlos formando gotas en el fondo de una caja de Petri pequeña. El extremo más delgado de cada pajilla OPS, fue cargado por capilaridad al tocar la superficie de una gota de solución de vitrificación más los oocitos. Las pajillas fueron llevadas directamente al nitrógeno líquido dentro de 25 segundos, para ser mantenidas allí hasta su devitrificación.

Para la devitrificación cada paj illa fue retirada desde el nitrógeno líquido y expuesta al aire por 3 segundos, para luego sumergir su extremo más delgado en $1.2 \mathrm{ml}$ de un primer medio de devitrificación (D1) compuesto por TCM-199 Hepes con $20 \%$ de SFB y $0.25 \mathrm{M}$ de sucrosa. Los oocitos fueron expelidos en el medio a $39^{\circ} \mathrm{C}$ durante 5 minutos. Después fueron transferidos a una segunda solución de desvitrificación (D2) compuesta por TCM-
199 Hepes con 0.15M de sucrosa, durante 5 minutos a $39^{\circ} \mathrm{C}$, para luego ser expuestos a solución EQ durante 5 minutos a 39으. Los oocitos fueron finalmente lavados en HepesTL suplementado, y destinados para la evaluación de su potencial de membrana mitocondrial.

\section{Determinación de la actividad mitocondrial de oocitos. Un total de 40} oocitos para cada tratamiento de maduración in vitro (con y sin suero), fueron cuantificados y evaluados individualmente para su porcentaje de fluorescencia. Sobre los oocitos madurados se utilizó el fluorocromo reportero de potencial de membrana interna mitocondrial JC-1 (5,5 6,6-tetracloro-1, 1,3,3yoduro de tetraetilbenzimidazolicarbocianina, Molecular Probes) a $1 \mathrm{\mu g} / \mathrm{ml}$ en medio de maduración, con incubación a $38.5^{\circ} \mathrm{C}$ durante 25 minutos. Los dímeros de J C- 1 formados a altos potenciales de membrana ( $>140 \mathrm{mV}$ ) fueron observados mediante microscopia de fluorescencia con un filtro de rodamina (máxima emisión 590 nm) BP 450-490, FT 510, LP 515, que muestra un color rojo. Se realizó fotografía digital (Sony DSC-S85) de las emisiones fluorescentes observadas en los oocitos, las cuales fueron cuantificadas en unidades relativas por su densidad media mediante el programa Scion Image Alpha 4.0.3.2 (Scion Corporation), y posteriormente transformadas a valores porcentuales con referencia a valores máximos de fluorescencia en células de la granulosa y mínimos en imágenes sin fluorescencia alguna. A través de una caracterización cualitativa de la fluorescencia, fueron determinadas las proporciones para los patrones de distribución de mitocondrias con alto potencial de membrana mitocondrial (fluorescencia roja), a partir de entre 40 y 71 oocitos desvitrificados derivados de ambos tratamientos de maduración, y para los grupos control respectivos de oocitos frescos (no criopreservados).

Análisis estadístico. El análisis estadístico incluyo una valoración descriptiva y la comparación de los grupos mediante una prueba $t$ de Student en lo relacionado con los porcentajes de fluorescencia (potencial de membrana mitocondrial) de oocitos individuales, y un análisis de varianza para 
la evaluación de los patrones de distribución de las mitocondrias de alto potencial de membrana, a partir de proporciones derivadas de unidades experimentales de 5 oocitos cada una. Todos los análisis fueron realizados mediante el programa Statgraphics Plus 3.0 (Statistical Graphics Corporation).

\section{RESULTADOS}

Cuantificación de la actividad mitocondrial. Los resultados obtenidos mostraron una diferencia estadísticamente significativa $(p<0.05)$ para el potencial de membrana mitocondrial de oocitos criopreservados (desvitrificados), entre los tratamientos de maduración in vitro. Se evidenció superioridad estadística en la actividad mitocondrial de los oocitos desvitrificados previamente madurados en un medio sin suero, respecto a los oocitos madurados en un medio con suero. En la tabla 1 se presentan los valores estadísticos para los resultados obtenidos.

Tabla 1. Valores estadísticos para el porcentaje de fluorescencia (potencial de membrana mitocondrial) de oocitos desvitrificados.

\begin{tabular}{lcc}
\hline Parámetro*/ & Sin Suero Con Suero \\
\hline Tratamiento & 40 & 40 \\
\hline Número de oocitos & $50.3^{\circ}$ & $39.8^{\circ}$ \\
Promedio & 16.4 & 15.1 \\
Desviación estándar & 2.59 & 2.38 \\
\hline
\end{tabular}

* Valores en unidades relativas (\% de fluorescencia). a,b Letras diferentes indican diferencia estadística significativa $(p<0.05)$ entre los tratamientos.

Se encontraron rangos de potencial de membrana mitocondrial (valores mínimos y máximos de fluorescencia) para oocitos desvitrificados previamente madurados con y sin suero de $11.3 \%$ a $70.6 \%$ y $18.8 \%$ a $83.1 \%$, respectivamente. Los valores de fluorescencia encontrados correspondieron a las proporciones de detección de mitocondrias con altos niveles de potencial de membrana mitocondrial ( $>140 \mathrm{mV}$ ). La figura 1 muestra oocitos desvitrificados de ambos tratamientos de maduración con diferentes niveles de potencial de membrana mitocondrial.

La figura 2 muestra un histograma comparativo de la distribución de los oocitos desvitrificados madurados por ambos tratamientos, según su
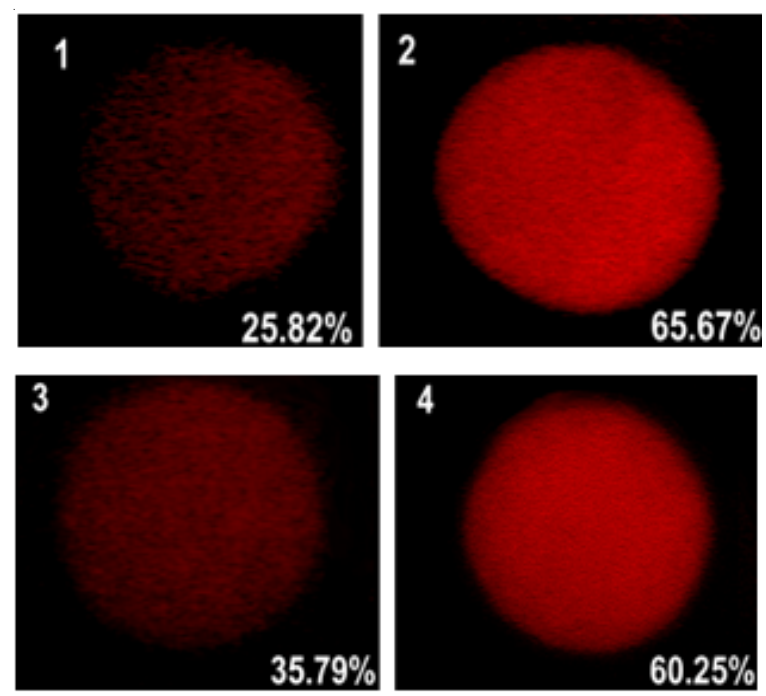

Figura 1. Oocitos evaluados para potencial de membrana mitocondrial (JC-1), con diferentes intensidades de fluorescencia expresado en porcentaje. 1 y 2. Oocitos desvitrificados madurados en medio sin suero; 3 y 4. oocitos desvitrificados madurados en medio con suero.

porcentaje de fluorescencia. La distribución de los niveles de potencial de membrana mitocondrial entre los dos tratamientos de maduración, mostró como para los oocitos desvitrificados previamente madurados en un medio sin suero se encontró una mayor proporción de oocitos en los rangos superiores de potencial de membrana mitocondrial expresada como porcentaje de fluorescencia, respecto a los oocitos madurados en un medio con suero.

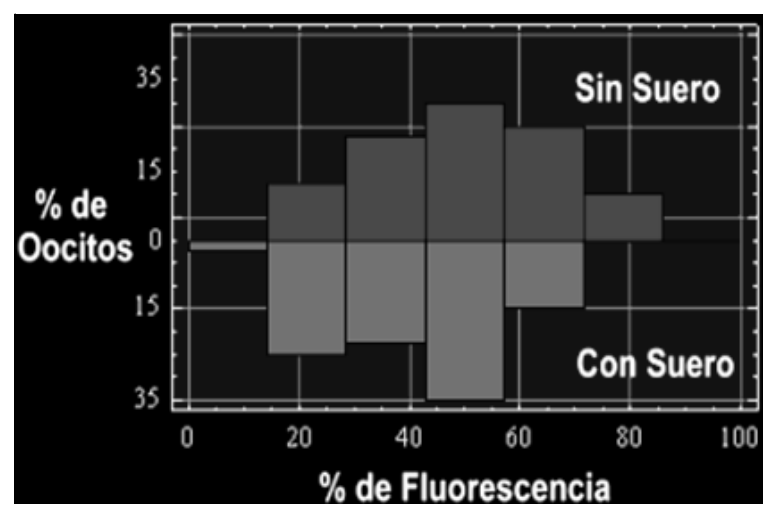

Figura 2. Histograma comparativo entre los porcentajes de fluorescencia (potencial de membrana mitocondrial) de oocitos desvitrificados madurados con y sin suero. 
Distribución de las mitocondrias con alta actividad mitocondrial. Respecto a la caracterización cualitativa de la distribución de la fluorescencia (asociada con el alto potencial de membrana mitocondrial) para los oocitos desvitrificados y los grupos control de oocitos frescos previamente madurados en tratamientos con y sin suero, se observaron los tipos de distribución difusa y pericitoplasmática (Figura 3). En la tabla 2 se reportan los resultados encontrados.
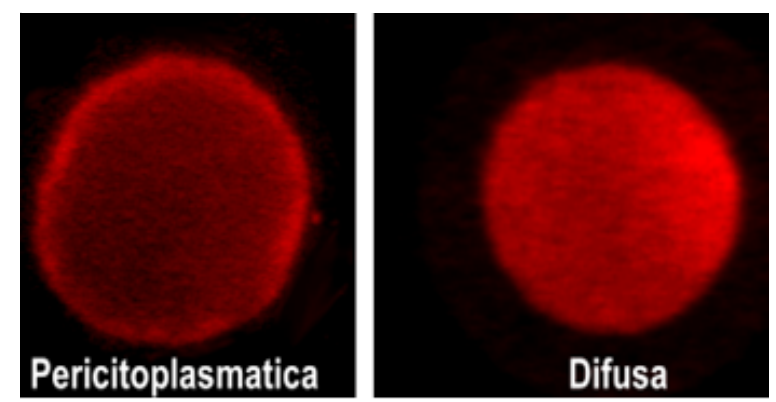

Figura 3. Oocitos con patrones de distribución difusa y pericitoplasmática de mitocondrias con alto potencial de membrana, detectado por fluorescencia de JC-1.

Los resultados no evidenciaron un efecto estadísticamente significativo $(p<0.05)$ del suero fetal bovino en la maduración in vitro, sobre la distribución de mitocondrias de alto potencial de membrana mitocondrial, tanto para oocitos frescos como para oocitos desvitrificados. Sin embargo, se evidenció una diferencia significativa $(p<0.05)$ para la distribución de mitocondrias de alto potencial de membrana, entre los oocitos frescos y desvitrificados para ambos tratamientos de maduración in vitro, lo que implica un efecto de la criopreservación sobre dicha distribución.

\section{SCUSI ÓN}

Los resultados obtenidos evidenciaron un efecto deletéreo de la presencia de suero fetal bovino en la maduración in vitro de oocitos bovinos sobre su actividad mitocondrial posterior a la criopreservación. De manera que la presencia de suero en este medio de cultivo, puede considerarse como responsable de conducir a un trastorno en el metabolismo mitocondrial de los oocitos criopreservados. Es factible considerar que la disminución en el potencial de membrana mitocondrial en oocitos madurados con suero, haya sido acentuada por el aumento en la sensibilidad a la criopreservación derivada del cultivo en presencia de dicho suplemento.

Dada la reconocida importancia de la actividad mitocondrial en el desarrollo embrionario, para el caso de los oocitos criopreservados el hecho de que su actividad mitocondrial se vea afectada negativamente por un cultivo previo en un medio con suero fetal, representa la posibilidad de que sus tasas de desarrollo in vitro se vean aún más limitadas de lo que son por el efecto de la criopreservación, lo cual es factible dado que en resultados preliminares a esta investigación, fue observado un efecto deletéreo de la presencia de suero fetal en la maduración in vitro de oocitos bovinos (no criopreservados) sobre su potencial de membrana mitocondrial, al igual que sobre su tasa de desarrollo embrionario.

Para el caso de la evaluación de los patrones de distribución de mitocondrias de alto potencial de membrana, en los grupos control

Tabla 2. Distribución de las mitocondrias con alto potencial de membrana en oocitos frescos (No crio) y desvitrificados (Crio), madurados con y sin suero.

\begin{tabular}{lcccc}
\hline & $\begin{array}{c}\text { Crio Con } \\
\text { suero }\end{array}$ & $\begin{array}{c}\text { Frescos Con } \\
\text { suero }\end{array}$ & $\begin{array}{c}\text { Crio Sin * Frescos Sin } \\
\text { suero }\end{array}$ & $\begin{array}{c}\text { * } \\
\text { suero }\end{array}$ \\
\hline Número de oocitos & 47 & 40 & 40 & 71 \\
Distribución difusa (\%) & $100^{\circ}$ & $80^{\circ}$ & $97.5^{\circ}$ & $85^{\circ}$ \\
Distribución pericitoplasmática (\%) & 0 & 20 & 2.5 & 15 \\
\hline
\end{tabular}

* Grupos control de oocitos frescos (no criopreservados) para cada tratamiento. a,betras diferentes indican diferencia estadística significativa $(p<0.05)$ entre los tratamientos. 
de oocitos frescos madurados con suero y sin suero se encontró un predominio de la distribución difusa, y se encontraron respectivamente proporciones del $20 \%$ y $15 \%$ de oocitos con distribución pericitoplasmática. Estos resultados son similares a los hallados por Tarazona et al (27), quienes encontraron que en oocitos frescos maduros, la distribución de mitocondrias es difusa y pericitoplasmática en proporciones del $87.3 \%$ y $12.7 \%$ respectivamente.

Los resultados no evidenciaron un efecto del suero sobre la actividad mitocondrial representada por la distribución de mitocondrias de alto potencial de membrana. Sin embargo, sí evidencian un efecto de la criopreservación sobre dicha distribución, ya que se presentó una disminución considerable en la proporción de distribución pericitoplasmática de mitocondrias de alto potencial de membrana para ambos tratamientos de maduración in vitro $(0 \mathrm{a}$ $2.5 \%)$ respecto a los oocitos no criopreservados (15 a $20 \%$ ), con el consecuente aumento en el patrón de distribución difusa. Estos resultados coinciden con los presentados por J ones et al (8) quienes reportaron para oocitos maduros humanos, pérdidas en la actividad mitocondrial a causa de la criopreservación, respecto a oocitos frescos. Considerando que la presencia de un patrón de distribución pericitoplasmático de mitocondrias de alto potencial de membrana ha sido asociada con mejores resultados en el desarrollo embrionario a partir de oocitos maduros, es posible que dicha variación en los patrones de distribución se refleje de manera negativa en las tasas de desarrollo de los oocitos criopreservados.

Es relevante la aplicación de un método de cuantificación de la fluorescencia detectada por JC-1 como reporte de la actividad mitocondrial, ya que generalmente los estudios se han basado en apreciaciones cualitativas de dicho parámetro, este metodo permite una evaluación más precisa y menos subjetiva de la actividad mitocondrial. El reporte de la alteración por la criopreservación de las proporciones de los diferentes patrones de distribución de mitocondrias de alto potencial de membrana en oocitos bovinos madurados, es importante ya que dicho fenómeno solo había sido descrito para oocitos humanos (8) y murinos (26).

De acuerdo con los resultados se concluye que bajo las condiciones de esta investigación, cuando se realiza la maduración in vitro de los oocitos bovinos en ausencia de suero fetal bovino, éstos presentan mayores proporciones de actividad mitocondrial después del proceso de criopreservación. Además, la criopreservación modifica las proporciones de los patrones de distribución de mitocondrias de alto potencial de membrana, de los oocitos bovinos madurados in vitro, respecto a aquellos oocitos madurados no criopreservados, conduciendo a la disminución en la proporción de distribución pericitoplasmática, con el consecuente aumento en la proporción de distribución difusa de mitocondrias con alto potencial de membrana.

\section{REFERENCIAS}

1. Albarracin MJ. Vitrificación de ovocitos bovinos mediante la técnica open pulled straw: estudio estructural de cromosomas microtúbulos y microfilamentos y posterior desarrollo embrionario in vitro. [Tesis doctoral]. España, Bellaterra: Universidad Autónoma de Barcelona, Facultad de Veterinaria; 2005.
2. Han B, Bischof J. Direct cell injury associated with eutectic crystallization during freezing. Cryobiology 2004; 48: 8-21. 
3. Mavrides A, Morroll D. Cryopreservation of bovine oocytes; is cryoloop vitrification the future to preserving the female gamete? Reprod Nut Dev 2002; 42: 73-80.

4. Picton H, Gosden $\mathrm{R}$, Leibo $\mathrm{S}$. Cryopreservation of oocytes and ovarian tissue: Gamete source manipulation and disposition. Geneva: WHO Headquarters (En linea) 2002 Septiembre 17 (Fecha de acceso 20 de Noviembre de 2008); URL disponible en: http://www.who.int/ reproductive-health/infertility/ report_content.htm

5. Chen S, Lien Y, Chao K, Ho H, Yang Y, Lee $\mathrm{T}$. Effects of cryopreservation on meiotic spindles of oocytes and its dynamics after thawing: Clinical implications in oocyte freezing - A review article. Mol Cell Endocrinol 2003; 202: 101-107.

6. Odani M, Komatsu Y, Oka S, I wahashi $\mathrm{H}$. Screening of genes that respond to cryopreservation stress using yeast DNA microarray. Cryobiology 2003; 47: 155-164.

7. Kathryn S, Parks J. Effects of cryopreservation procedures on the cytology and fertilization rate of in vitromatured bovine oocytes. Biol Reprod 1999; 61: 178-187.

8. Jones A, Van Blerkom J, Davis, Toledo A. Cryopreservation of metaphase II human oocytes effects mitochondrial membrane potential: Implications for developmental competence. Hum Reprod 2004; 19(8): 1861-1866.

9. Paynter S. Current status of the cryopreservation of human unfertilized oocytes. Hum Reprod Update 2000; 6(5): 449-456.

10. Hyttel P, Vajta G, Callesen H. Vitrification de bovine oocytes with the open pulled straw method: Ultrastructural consequences. Mol Reprod Dev 2000; 56(1): 80-88.
11. Leroy J, Genicot G, Donnay I, Van Soom A. Evaluation of the lipid content in bovine oocytes and embryos with nile red: a practical approach. Reprod Domest Anim 2005; 40: 76-78.

12. Mucci N, Aller J, Kaiser G, Hozbor F, Albeiro R. Producción in vitro de embriones bovinos: suplementación de los medios de cultivo con suero. Arch Med Vet 2006; 38(2): 97-104.

13. Ferguson E, Leese $H$. Tryglyceride content of bovine oocytes and early embryos. J Rep Fertil 1999; 116: 373-378.

14. Massip A. Cryopreservation of bovine oocytes: current status and recent developments, minireview. Reprod Nut Dev 2003; 43: 325-330.

15. Shaw J, Jones G. Terminology associated with vitrification and other cryopreservation procedures for oocytes and embryos. Hum Reprod Update 2003; 9(6): 583-605.

16. Abe $\mathrm{H}$, Hoshi $\mathrm{H}$. Evaluation of bovine embryos produced in high performance serum free media. J Reprod Dev 2003; 49(3): 193-202.

17. Kim J, Kinoshita M, Ohnishi M, Fukui P. Lipid and fatty acid analysis of fresh and frozen-thawed immature and in vitro matured bovine oocytes. Reproduction 2001; 122: 131-138.

18. Bavister B, Squirrell J. Mitochondrial distribution and function in oocytes and early embryos. Hum Reprod 2000; 15(suppl 2): 189-198.

19. Wilding $M$, Dale $B$, Marino $M$, di Mateo $L$, Alvigg $C$, Pisaturo $M$ et al. Mitochondrial aggregation patterns and activity in human oocytes and preimplantation embryos. Hum Reprod 2001; 16(5): 909-917. 
20. Van Blerkom J, Davis $\mathrm{P}$, Alexander S. Inner mitochondrial membrane potential, cytoplasmatic ATP content and free $\mathrm{Ca}(2+)$ levels in metaphase II mouse oocytes. Hum Reprod 2003; 17(2): 393-406.

21. Wilding $M$, De Placido $G$, De Matteo $L$, Marino $M$, Alviggi $C$, et al. Chaotic mosaicism in human preimplantation embryos is correlated with a low mitochondrial membrane potential. Fertil Steril 2003; 79: 340-346.

22. Acton B, Jurisicova A, Jurisica I, Carper R. Alterations in mitochondrial membrane potential during preimplantation stages of mouse and human development. Mol Hum Reprod 2004; 10: 23-32.

23. Au H, Yeh T, Kao S, Tzeng C, Hsieh R. Abnormal mitochondrial structure in human unfertilized oocytes and arrested embryos. Annals of the New York Academy of Sciences 2005; 1042: 177-185.

24. Van Blerkom J. Mitochondria in human oogenesis and preimplantation embryogenesis: engines of metabolism, ionic regulation and developmental competence. Reproduction 2004; 128 : 269-280.
25. Hasler J. In-vitro production of cattle embryos; problems with pregnancies and parturition. Hum Reprod 2000; 15(5): 47-58.

26. Ahn J, Sohn I, Kwon H, J O D, Park Y, Churl M. Characteristics of the cell membrane fluidity, actin fibers, and mitochondrial dysfunctions of frozenthawed two-cell mouse embryos. Mol Reprod Dev 2002; 61: 466-476.

27. Tarazona A, Rodríguez J, Restrepo $L$, Olivera-Angel M. Mitochondrial activity, distribution and segregation in bovine oocytes and in embryos produced in vitro. Reprod Domest Anim 2006; 41(1): 5-11.

28. Brevini $T$, Vassena $R$, Francisci $C$, Gandolfi $F$. Role of adenosine triphosphate active mitochondria and microtubules in the acquisition of developmental competence of parthenogenetically activated pig oocytes. Biol Reprod 2005; 72: 1218-1223.

29. Krisher R. The effect of oocyte quality on development. J Anim Sci 2004; 82 (E Suppl): E14-E23.

30. Vajta G, Holm P, Kawayama M, Booth $P$, Jacobsen $\mathrm{H}$, Greve $\mathrm{T}$, Callesen $\mathrm{H}$. Open pulled straw vitrification: A new way to reduce cryoinjuries of bovine ova and embryos. Mol Reprod Dev 1998; 51(1): 53-58. 